\title{
HACIA UN CONCEPTO DE CIENCIA INTERCULTURAL
}

\author{
Cabo Her nández, José Manuel ${ }^{1}$ y Enrique Mirón, Car me ${ }^{2}$ \\ ${ }^{1}$ Departamento de Didáctica de las Ciencias Experimentales. Facultad de Educación. Campus de Melilla. Universidad de Granada \\ ${ }^{2}$ Departamento de Química Inorgánica. Facultad de Educación. Campus de Melilla \\ jmcabo@ugr.es \\ cenrique@ugr.es
}

\begin{abstract}
Resumen. Se presenta una revisión extensa de bibliografía sobre ciencia intercultural. Este tópico ha provocado en la pasada década un animado debate en la comunidad internacional que se dedica a la investigación sobre la enseñanza de las ciencias, especialmente en la cultura anglosajona. La escasez de trabajos publicados en España sobre ciencia intercultural frente a la importante diversidad cultural existente en nuestro país, con el añadido del reciente fenómeno de la inmigración, recomiendan la revisión del tópico y la promoción de investigaciones en este ámbito de trabajo. Se analizan las líneas existentes y los debates suscitados, relacionándolos como un tópico integrado en los enfoques ciencia-tecnología-sociedad.

Palabras clave. Ciencia-tecnología-sociedad, ciencia intercultural, educación intercultural, enseñanza de las ciencias.
\end{abstract}

Summary. An extensive review of the bibliography on intercultural science is presented. In the past decade, this topic has provoked a lively debate within the international community, which is committed to the study of science education, especially in Anglo-Saxon culture. The shortage of works published in Spain on intercultural science, as compared to the important cultural diversity existing in our country, together with the addition of the recent phenomenon of immigration, calls for the revision of the topic and the promotion of investigation in this line of work. The existing outlines and resulting debates are analysed, relating them as an integrated topic within the science-technology-society fields.

Keywords. Science-technology-society, intercultural science, intercultural education, science education.

\section{INTRODUCCIÓN}

El desarrollo de la educación intercultural en Europa ha estado vinculado en gran medida a los fenómenos de inmigración. El impacto social de la inmigración en España es posterior al resto de Europa, pues históricamente somos un país de emigración y por ello el desarrollo de la educación intercultural es más reciente en nuestro país (Aguado y Malik, 2001). La investigación sobre educación intercultural en Europa (Bartolomé, 1997), en sus líneas generales, mantiene su interés en problemáticas generales como las derivadas de las lenguas o las actitudes hacia las diferencias culturales como forma de prevención del racismo y la xenofobia, sin entrar decididamente en problemas que afectan a contenidos específicos del currículo. Todo ello contribuye a que la ciencia de orientación multicultural no sea prioritaria en nuestro contexto.
Frente a la tradición europea, existen otros enfoques en educación intercultural. En concreto, en países desarrollados de habla inglesa (EEUU, Canadá, Australia, Nueva Zelanda y Reino Unido) se trabaja desde hace varias décadas en el contexto multicultural, lo que ha facilitado la aparición de un buen número de trabajos, especialmente a partir de la década de los noventa, que se han planteado la intercultura en las clases de ciencias. Podríamos hablar, por tanto, de una tradición anglosajona y otra europea en este sentido, al igual que se plantea en el campo CTS (González, López y Luján, 1996), sin menospreciar propuestas de síntesis, especialmente las desarrolladas desde la OEI (Organización de Estados Iberoamericanos) en cuyos países coexiste una rica variedad de culturas indígenas. 
El contexto escolar multicultural que se presenta en España, véase el caso de algunas comarcas de Almería (Soriano, 1997), es la escolarización de alumnado inmigrante en situación de minorías que en general no conoce la lengua escolar y viven en un entorno socioeconómico deprimido, por lo que los problemas prioritarios se centran en el dominio de la lengua y la integración social más que en el aprendizaje de contenidos específicos. Sin embargo, existen otros contextos multiculturales españoles: los casos de las ciudades autónomas de Ceuta y Melilla, la problemática educativa del pueblo gitano o la diversidad cultural que se desprende de la estructura autonómica española (Muñoz, 1997).

El retraso apuntado por Aguado y Malik (2001) para el campo general de la educación intercultural en España se confirma para la ciencia intercultural por la escasez de trabajos publicados (Membiela, 1999; Benarroch, 2001), retraso que es general en el ámbito internacional (Krugly-Smolska, 1996, 1997). Sin embargo, en la última década, creemos que existe, en la comunidad internacional que trabaja en didáctica de las ciencias, un importante debate sobre ciencia intercultural que afecta al núcleo central de nuestro trabajo, especialmente en el mundo anglosajón.

\section{OBJETIVOS}

Pretendemos en este trabajo realizar una revisión bibliográfica que nos permita describir los aspectos básicos sometidos a discusión sobre el concepto de ciencia intercultural en el ámbito de la didáctica de las ciencias experimentales y cotejarlos con trabajos compiladores sobre educación intercultural con la finalidad de establecer un panorama general contextualizado de esta actividad de investigación, aunque, dada la complejidad de la cuestión, cada una de las temáticas implicadas tenga suficiente trascendencia como para ser tratada de forma independiente. En definitiva, perseguimos cuatro objetivos básicos:

1) Delimitar el ámbito de trabajo de la ciencia intercultural y describir los paradigmas alternativos que sostienen las diversas propuestas de actuación en este campo, identificando los núcleos centrales del debate.

2) Determinar cuáles han sido las líneas de investigación desarrolladas en ciencia intercultural hasta el presente, especialmente en el contexto cultural anglosajón.

3) Establecer comparaciones entre las líneas desarrolladas en educación intercultural y las específicas de ciencia intercultural.

4) Defender las aportaciones de la ciencia intercultural en el conjunto de preocupaciones que guían la investigación en didáctica de las ciencias experimentales al considerar que su papel no es o no debe ser marginal en el conjunto de nuestro ámbito de trabajo.

\section{DELIMITACIÓN Y EVOLUCIÓN DEL ÁMBITO DE TRABAJO DE CIENCIA INTERCULTURAL}

Debemos señalar una cierta confusión debida a la polisemia en el uso de los términos multicultural e intercultural. En el presente trabajo adoptamos las siguientes precisiones terminológicas. Los términos traducidos del inglés transcultural, multiétnico y multicultural se usan como sinónimos. Para nosotros, multicultural tiene que ver con el contexto escolar y social, es decir, se trata de la presencia en las aulas y en la sociedad de personas de distintas culturas de origen. Educación intercultural tiene que ver con los cambios o reformas necesarios en los sistemas educativos ante la existencia de un contexto multicultural. Por tanto, entendemos por ciencia intercultural al conjunto de cambios y reformas curriculares que se deben realizar en el contexto educativo de las ciencias experimentales para responder a la existencia de un contexto multicultural.

Tras la identificación del nuevo reto, el siguiente paso lógico era establecer una agenda de trabajo o catálogo de actuaciones prioritarias para la investigación en didáctica de las ciencias. Los trabajos presentados por Aikenhead (1993) en la revista Science Education en su monográfico sobre ciencia intercultural tuvieron la virtud de iniciar un debate que se continúa y amplía actualmente.

Rakow y Bermúdez (1993) centran el problema en los bajos rendimientos y el abandono de estudiantes hispanos en EEUU en los estudios de ciencias y desarrollan propuestas de actuación. En cambio, el resto de autores propone agendas de trabajo más generales y no vinculadas a un problema específico con distinto grado de radicalidad (Atwater y Riley, 1993; Hodson, 1993).

Tras la publicación de estas propuestas, aparece un controvertido trabajo, el de Stanley y Brickhouse (1994), señalando que la ciencia intercultural responde básicamente a dos retos: el acceso de las minorías y las mujeres a los estudios de ciencias, siguiendo una orientación de ciencias para todos; y cambios sobre qué ciencia enseñar, incluyendo la propia naturaleza epistemológica de la ciencia. Consideran que este segundo reto no ha sido suficientemente desarrollado y profundizan en esta segunda cuestión presentando la ciencia desde dos orientaciones: la universalista y la multiculturalista.

La orientación universalista de ciencia, de claro signo positivista, defiende la independencia de ésta de los intereses humanos, cultura, género, raza, clase, etnia y orientación sexual, asumiendo que lo que llamamos ciencia es la única manera de obtener conocimiento válido frente a otras orientaciones culturales que pondrían en tela de juicio la objetividad de ésta.

La perspectiva multiculturalista aportaría ideas y conceptos de Kuhn y Lakatos, según los cuales es fundamental incorporar varias posibilidades para la comprensión y producción de conocimiento. La diversidad de perspectivas no asegura la fiabilidad del conocimiento pero crea las condiciones requeridas para el progreso científico. De esta manera, la ciencia estaría influida por 
factores culturales como los relacionados anteriormente. La historia de la ciencia demuestra que los propósitos, teorías y metodologías han cambiado como consecuencia de grandes cambios culturales. La perspectiva occidental es una vía válida para producir conocimiento pero no la única, ya que la perspectiva multicultural acepta la posibilidad de que conocimientos producidos por tradiciones culturales diferentes a la cultura occidental sean igualmente válidos, por ejemplo, en el campo de la farmacología con la fitoterapia tradicional.

Desde la publicación del trabajo de Stanley y Brickhouse hasta 1996 aparecieron sucesivas críticas hacia la perspectiva multiculturalista y hacia las formas políticamente más comprometidas de ciencia intercultural, como el planteamiento antirracista de Hodson.

Williams (1994) critica a Hodson; y Good (1995), Loving (1995) y Ahlgren (1996) critican a Stanley y Brickhouse.

El cruce de argumentaciones es interesante, pues centra la atención sobre aquellos aspectos que la comunidad científica no ha consensuado y permite identificar los núcleos centrales del debate.

Williams plantea el problema del relativismo cultural que achaca a Hodson. Ante la aceptación y respeto hacia culturas no occidentales recuerda la existencia de prácticas sociales no admisibles como el papel de la mujer en el islam. Además, recuerda que las posiciones antirracistas pueden provocar el efecto contrario al buscado, provocando el rechazo hacia la ciencia en alumnado de culturas no occidentales.

Good y Ahlgren salen en defensa de la American Association for the Advancement of Science (AAAS) y de sus propuestas curriculares, que son criticadas desde la perspectiva multicultural, coincidiendo parcialmente con Loving en cuanto a los significados atribuidos al concepto universalista de ciencia. Argumentan, por ejemplo, que científicos de todas las culturas han realizado aportaciones a la ciencia y recuerdan que los documentos de la AAAS reconocen la importancia de factores culturales en ciencias como género y etnia. Loving matiza la diferencia entre la perspectiva multicultural y la perspectiva pluralista, que considera propia de la ciencia en el sentido universalista, al tiempo que afirma encontrar distintos grados de radicalidad en los discursos a favor de la ciencia multicultural.

Pero no todo han sido críticas hacia los enfoques de ciencia intercultural. La discriminación entre ciencia personal o ciencia «indígena», ciencia escolar y ciencia occidental también ha provocado nuevos trabajos (Ogawa, 1995) y un cierto desarrollo de la agenda propuesta por los defensores de la ciencia intercultural, apoyada en parte por los estudios sociales sobre ciencia y tecnología, especialmente por la identificación de un modelo de ciencia como práctica sociocultural (Callon, 2001) o como enculturación de prácticas y habilidades.

De hecho, el cambio de milenio ha supuesto un aumento en la aparición de trabajos sobre ciencia intercultural. En 1998 se organiza un simposium sobre multiculturalismo en la National Association for Research in Science Teachining Annual Meeting, cuyas ponencias plenarias y discusiones aparecen más tarde en forma de número monográfico de la revista Science Education (Vol. 85, 1).

En el año 2001 aparece, en la revista Journal of Research in Science Teaching el volumen 38 (5) dedicado por entero a la lengua y cultura en educación de ciencias. En la presentación del número, Lee (2001) justifica el contenido del monográfico por la necesidad creciente de igualdad para una población escolar cada vez más diversa cultural y lingüísticamente, lo que afecta a preguntas tales como qué es la ciencia, qué contenidos deberían ser enseñados, cómo deberían ser enseñados y cómo deberían ser evaluados los aprendizajes de los alumnos. Tras este monográfico, la misma revista publicó tres números el mismo año (Vol. 38 [8, 9, 10]) dedicados al contexto urbano de la enseñanza de las ciencias.

Las revisiones sobre ciencia intercultural que estos monográficos proponen ponen de manifiesto una continuación de los núcleos centrales del debate iniciado en la década de los noventa. El concepto de ciencia para todos como idea central de la reforma de la enseñanza de las ciencias tiene más de una década. Sin embargo, esta idea ha fracasado en su aplicación ante los retos planteados por un alumnado cada vez más diverso cultural y lingüísticamente según Lynch (2001). Estos planteamientos muestran que el principio de igualdad de oportunidades y justicia social sigue siendo uno de los núcleos centrales de la ciencia intercultural, pues la tendencia social hacia el multiculturalismo continúa aumentando en todos los países del mundo.

El concepto alternativo de ciencia multiculturalista expuesto por Stanley y Brickhouse $(1994,2001)$ sigue recibiendo apoyos (Lewis y Aikenhead, 2001; BrownAcquaye, 2001) y críticas (Ortiz de Montellano, 2001; Irzik, 2001; Svennbeck, 2001), a veces de tipo pragmático, al entender que el profesorado de ciencias está muy lejos de la orientación multiculturalista (McKinley, 2001).

La introducción de contenidos tradicionales (ciencia indígena) en el currículo de ciencias, demasiado vaga y general, se ejemplifica en el llamado conocimiento ecológico tradicional (Snively y Corsiglia, 2001), que para estos autores debe incluirse en el currículo científico como parte de la ciencia escolar. Ahora bien, Cobern y Loving (2001) matizan la inclusión de los conocimientos tradicionales aceptando su inclusión desde el pluralismo epistemológico, es decir, discriminando entre las posiciones epistemológicas de la ciencia occidental y la ciencia personal.

\section{LÍNEAS DE INVESTIGACIÓN EN CIENCIA INTERCULTURAL}

Establecido el debate sobre las orientaciones universalistas y multiculturalistas y sobre el principio de igualdad de oportunidades, aparecen trabajos que desarrollan las propuestas de las respectivas agendas. Los trabajos consultados aquí se refieren a revistas específicas de didác- 
tica de las ciencias experimentales extranjeras (Science Education; Journal of Research in Science Teaching; Science \& Education; International Journal of Science Education...), dada la escasez de trabajos en revistas españolas (Membiela, 1999; Benarroch, 2001). La complejidad y variedad de las investigaciones aparecidas dificultan, por razones de espacio, una sistematización de todas y cada una de las líneas detectadas. En este sentido, y sólo teniendo en cuenta los contenidos de los problemas a resolver, podemos enumerar los siguientes bloques de trabajos:

A. Trabajos que muestran el multiculturalismo como una alternativa a las concepciones tradicionales de ciencia y educación en donde se pueden distinguir las dos grandes temáticas citadas: la igualdad de oportunidades de poblaciones culturalmente diversas y el debate no consensuado sobre la naturaleza epistemológica de la ciencia.

$B$. Trabajos que aportan una base teórica y empírica sobre la influencia de factores culturales en la ciencia, desarrollando un modelo de ciencia como práctica sociocultural, con aportaciones externas procedentes de sociología, antropología, historia, filosofía, y en definitiva, de los estudios sociales sobre ciencia y tecnología que sirven de apoyo a tesis defendidas sobre ciencia intercultural.

C. Trabajos sobre género y ciencia: los estudios de las mujeres y de género constituyen un movimiento emergente. Muchos trabajos de didáctica de las ciencias han identificado diferencias de género al evaluar diversos aspectos, tales como el rendimiento académico de las mujeres en las clases de ciencias y su menor presencia en las mismas (Manassero y Vázquez, 2003), las actitudes de las mujeres hacia la ciencia y la enseñanza de la ciencia (Farenga y Joyce, 1999; Jayaratne, Thomas y Trantmann, 2003) o hacia la relación entre actitudes y conocimientos (Mattern y Schau, 2002). Especialmente significativos son los trabajos sobre género que tienen su origen social en el movimiento feminista en sus diversas versiones (feminismo de igualdad, feminismo de diferencia...), tanto en los estudios sociales sobre ciencia y tecnología (González, 2001) como en el campo de la educación (Davis, 2001; 2002) y que no hemos tratado aquí por considerar que tienen un origen independiente y anterior a la preocupación multicultural, aunque exista una convergencia en estos estudios hacia la integración y consideración de un conjunto de variables como causas potenciales de discriminación, como son, además del género, la cultura, la etnia y la lengua (Chinn, 2002). El modelo de ciencia como práctica sociocultural abre las puertas hacia la convergencia de tendencias críticas, entendiendo las diferencias de género como «subcultura humana» transversal presente en todas las identidades culturales y sociales. Aparecen trabajos que analizan conjuntamente género y etnia, como los de Bianchini, Hilton-Brown y Breton (2002), Bianchini y Solomon (2003).

Por razones de espacio, desarrollamos en este trabajo sólo el bloque A. Los bloques B y C, dada su trascendencia, creemos que merecen un análisis independiente que podría dar pie a otras revisiones exhaustivas en cada uno de los campos a los que hacen referencia.

Para sintetizar las investigaciones en ciencia intercultural aludidas en el apartado A, las hemos agrupado en siete temáticas concretas, que no presentan un equilibrio cuantitativo en cuanto a trabajos pero que marcan claramente posibles líneas de investigación diferenciadas, aunque el esquema de análisis seguido, en base a los problemas tratados, sea meramente tentativo de una realidad mucho más compleja en la que se pueden identificar investigaciones que pertenecen simultáneamente a varias de las líneas descritas. Las siete temáticas referidas son las siguientes:

\section{A. 1. Igualdad de oportunidades}

Junto a propuestas radicales que explicitan el carácter sociopolítico de toda enseñanza de ciencias (Hodson, 1999), el principio de igualdad de oportunidades ha seguido orientando trabajos hacia el análisis de las causas del mayor fracaso escolar y del mayor abandono de los cursos de ciencias en minorías culturales, especialmente en EEUU. Atwater, Wiggins y Gardner (1995) encuentran baja autoestima y actitudes negativas hacia la ciencia en alumnado de minorías culturales, y Barton y Yang (2000) presentan un estudio de caso con un alumno hispano que vive las clases de ciencias como un fenómeno de asimilación cultural. Ya señalamos el caso de Rakow y Bermúdez (1993) con la minoría hispana, y de Rodríguez (1997, 1998). Estos trabajos se extienden a las minorías asiáticas (Lee, 1997) y negras (Atwater, 2000).

\section{A. 2. Desarrollo del currículo de ciencias en países y} alumnado de cultura no occidental

Una de las tendencias más desarrolladas de las agendas multiculturales es la contextualización de los currículos de ciencias en países de cultura no occidental o de minorías culturales en países occidentales (Baker y Taylor, 1995; Cobern, 1996; Krugly-Smolska, 1995). Se defienden programas biculturales orientados a establecer puentes cognitivos entre culturas (Aikenhead y Jegede, 1999; Aikenhead, 2001), que se aplican en culturas indígenas en EEUU (Allen y Crawley, 1998) y Canadá (Aikenhead, 1997). Y se estudian casos concretos de distinto ámbito geográfico, ya sea a escala continental en el caso africano (Jegede, 1997); o en contextos específicos, como Botswana (Ogunniyi, 1995), Swazilandia (Lubben, Campbell y Dlamini, 1996); o con características específicas, como el uso tradicional de metáforas (Lubben, Netshisaulu y Campbell, 1999) o el tiempo de espera en preguntas de clase (Jegede y Olajide, 1995). Rennie y Dunne (1994) exploran las percepciones acerca de la ciencia en Fiji, incluyendo la variable género. Waldrip y Taylor (1999a, 1999b) indagan sobre la contextualización del currículo de ciencias en Melanesia y Tsai (1998), de Taiwan. Akatugha y Wallace (1999) estudian la influencia cultural sobre el razonamiento proporcional de alumnado de física en Nigeria, mientras que Smith y Hausafus (1998) exploran la relación entre el rendimiento académico en ciencias y el interés de las familias de minorías por estos estudios. Zembylas (2002) identifica 
tensión entre los valores locales y las tendencias globales en el currículo científico en Chipre.

A. 3. Problemas de aprendizaje de ciencias centrados en la lengua, tanto por las diferencias entre la lengua materna y la lengua escolar, como por las singularidades del lenguaje científico.

Las diferencias entre la lengua materna y la lengua escolar es un claro campo de preocupación. Aunque la importancia de la lengua para el aprendizaje de las ciencias no esté ligada necesariamente al contexto multicultural (Lemke, 1997; Roth y Welzel, 2000; Unsworth, 2001), la perspectiva sociocultural la integra en la problemática (Lemke, 2001; Roth y Lawless, 2002). La lengua se ha identificado como una de las causas principales de fracaso escolar en las clases de ciencias (Feltham y Downs, 2002) y se ha tratado su influencia en el diagnóstico de concepciones (Clerk y Rutherford, 2000), encontrando diferencias en función de la lengua y cultura, en el alumnado nativo de Canadá, sobre percepciones de la naturaleza de la ciencia (Sutherland y Dennick, 2002). Además, Lynch (1996) encuentra pruebas de que algunos esquemas alternativos sobre los conceptos de gas, sólido y líquido pueden ser determinados lingüística y culturalmente en muestras de alumnado de Filipinas y de Tasmania. Por otro lado, se han utilizado herramientas semióticas para diseñar actividades en mexicanos escolarizados en EEUU (Duran, Dugan y Weffer, 1998), mientras que Kearsey y Turner (1999) encuentran ventajas en alumnado bilingüe para la comprensión de lenguaje científico

A. 4. Interferencias entre ciencia y religión, más significativas en el contexto americano que europeo

En trabajos anteriores hemos revisado este ámbito de trabajo en donde se recogen algunas decenas de referencias (Cabo y Enrique, 2001; Cabo y Enrique, 2003; Enrique y Cabo, en prensa). Existen dos problemáticas concretas que interesa destacar aquí en relación con la ciencia intercultural: a) Por un lado, la existencia de conflictos entre ciencia y religión derivados especialmente de las respectivas naturalezas epistemológicas que influyen en las creencias personales del alumnado y en la imagen de la ciencia; y b) por otro lado, las interferencias de las creencias religiosas en la construcción de conceptos científicos, especialmente de aquéllos que tienen implicaciones personales y afectivas, como, por ejemplo, el origen de la especie humana y la teoría de la evolución. Consideramos la religión como parte de la cultura humana, y una de las características de la multiculturalidad es la convivencia en el aula de alumnado que profesa religiones diferentes junto con posiciones agnósticas o deístas no vinculadas a instituciones religiosas. Ello justifica para nosotros su inclusión aquí.

A. 5. Papel de los conocimientos tradicionales como parte de la ciencia personal (o ciencia indígena) y su inclusión en el currículo científico.

Algunos trabajos dirigen sus esfuerzos hacia el papel de las culturas indígenas en el currículo de ciencias (Ninnes, 2000; Kawagley, Norris-Tull y Norris-Tull, 1998), además de los ya citados (Snively y Corsiglia, 2001; Cobern y Loving, 2001).
A. 6. Revisión de libros de texto analizando culturalmente su contenido.

Eide y Heikkinen (1998) analizan los contenidos multiculturales de manuales de ciencias para profesores, encontrando que sólo el 11,7\% de los contenidos son muy relevantes desde el punto de vista cultural.

A. 7. Formación de profesorado en el contexto multicultural.

Luft, Bragg y Peters (1999) analizan las vivencias de una profesora en un contexto multicultural para identificar sus dificultades y sacar consecuencias sobre la formación inicial. Cabo y Enrique (en prensa) tratan estudios de casos en la Facultad de Educación del Campus de Melilla en maestros en formación, obteniendo indicios de interferencias entre ciencia y religión en el $50 \%$ de los casos.

\section{RELACIÓN ENTRE LA EDUCACIÓN INTER- CULTURAL Y LA CIENCIA INTERCULTURAL}

Los manuales generales sobre educación intercultural consultados (McCarthy, 1994; Muñoz, 1997; Sales y García, 1997) describen un panorama variado y complejo de programas sobre educación intercultural. $\mathrm{Nu}-$ merosos autores se afanan en clasificar y sistematizar las líneas realmente existentes, en función de uno u otro criterio. Enumeramos a modo de ejemplo una de las clasificaciones citada por Muñoz (1997):

1) Aditividad étnica: incorporación de contenidos étnicos al currículo escolar.

2) Desarrollo del autoconcepto de los alumnos de minorías étnicas.

3) Compensación de privaciones culturales.

4) Enseñanza de las lenguas de origen.

5) Lucha contra el racismo.

6) Crítica radical que busca la reforma de la estructura social.

7) Remedios para las dificultades genéticas.

8) Promoción del pluralismo cultural.

9) Diferencia cultural: programas educativos que incorporan las diversas culturas y sus estilos de aprendizaje.

10) Asimilación de los estudiantes en la cultura mayoritaria.

Algunos de los programas enunciados están dirigidos hacia la formación de minorías culturales y, por tanto, afectan a centros y aulas que presentan una diversidad cultural importante. En cambio, otros están dirigidos a todas las aulas y a todo el alumnado. Pero, si en algo co- 
inciden estos autores, es en relacionar los programas interculturales con las posturas ideológicas desde donde se diseñan las políticas educativas interculturales. En este sentido McCarthy (1994) identifica y relaciona los modelos de intervención en los que se basan los programas con la ideología política que la sustenta (conservadora, liberal, neomarxista, radical...).

La bibliografía consultada permite identificar tres modelos diferenciados de ciencia intercultural presentes en el ámbito general de la educación intercultural. En realidad, se trata de tres respuestas distintas al contexto multicultural frente a la posición tradicional, que niega la influencia de la cultura en la ciencia y la existencia de otras ciencias que no cumplan los estándares científicos convencionales. Esta posición sería criticada por la ciencia intercultural por asimilacionista y, aunque supone en sí misma una toma de posición ante la realidad multicultural, no la tenemos aquí en cuenta.

A. Modelos de competencia cultural: el profesorado debe ayudar a la identidad étnica, al conocimiento sobre los distintos grupos culturales y a la competencia en más de un sistema cultural. Un defensor de este modelo sería, por ejemplo, Aikenhead (2001). Las iniciativas van encaminadas hacia programas biculturales (ciencia personal o indígena frente a la ciencia occidental), estableciendo puentes cognitivos entre ambas ciencias. Se entiende que la adquisición de ciencia occidental puede ser un proceso de asimilación cultural al implicar el abandono de la cultura de origen si se parte del punto de vista de que el único conocimiento válido es el científico y la cultura del que aprende no es occidental, o sí lo es pero con una actitud crítica, por ejemplo, ante las consecuencias ambientales del desarrollo económico, incluyendo buena parte de la comunidad científica mundial.

$B$. Modelos de emancipación cultural (Seiler, Tobin y Sokolic, 2001): relaciona el éxito o fracaso escolar con la igualdad de oportunidades y la promoción social en el mundo laboral. El problema es la exclusión de las culturas minoritarias del currículo (de ciencias) y su baja autoestima.

$C$. Modelos de educación antirracista y de acción sociopolítica: propuestas radicales que expresan la necesidad de un papel activo y explícito hacia toda forma de racismo, opresión o discriminación de grupos humanos. Podemos citar, en este caso, a Hodson (1999).

Algunas propuestas son críticas con la educación intercultural y, por tanto, con la ciencia intercultural. De acuerdo con algunos teóricos (McCarthy, 1994), se observa una tendencia a relacionar, en el conjunto de influencias culturales, al género y la clase social junto con la identidad cultural. Raza, género y clase social serían tres variables interconectadas que se relacionan desde un punto de vista sistémico y que no pueden entenderse por separado de forma sumativa. En nuestro contexto específico de escolarización de alumnado musulmán en Melilla, por ejemplo, existen indicios, por observación de casos, de que las expectativas hacia la escolarización de musulmanes y musulmanas pueden ser muy diferentes, provocando diversas pautas de conductas. En general, cuando el profesorado se queja, por ejemplo, de las respuestas violentas y disruptivas en aulas de secundaria, se refiere en realidad al $50 \%$ potencial de su alumnado, es decir, a los alumnos. Esto nos lleva a una de las críticas de McCarthy al multiculturalismo al defender la heterogeneidad de todas las culturas, por la cual no podemos afirmar que las comunidades culturales al completo defiendan los mismos valores.

Las críticas hacia las diversas versiones de ciencia intercultural se basan, en el caso de las propuestas antirracistas, en que podrían obtener respuestas contrarias a lo pretendido, es decir, promover actitudes negativas hacia la ciencia por su insensibilidad a las diferencias culturales. Una segunda crítica abunda en el relativismo cultural. No todas las prácticas culturales, por ser diferentes, deben respetarse. Ya hemos señalado un ejemplo explícito de esta cuestión al referirnos al papel de la mujer en el islam. En cuanto al modelo de ciencia subyacente, no hay consenso en la comunidad científica hacia qué es la ciencia. Es más, aunque el modelo de ciencia como práctica sociocultural es defendido desde los estudios sociales de ciencia y tecnología y desde la orientación CTS en educación, los trabajos sobre las creencias epistemológicas del profesorado ponen de manifiesto la pervivencia del empirismo en el profesorado y en buena parte de la comunidad científica, lo que nos lleva a la importancia de nuevos planteamientos multiculturales en la formación del profesorado (inicial y permanente) para innovar en esta línea. En palabras de Porlán, Rivero y Martín (2000): «la visión del conocimiento científico como algo absoluto, objetivo, acabado, descontextualizado y neutral es el obstáculo epistemológico».

La principal iniciativa institucional del sistema educativo español hacia el contexto multicultural han sido los programas de compensatoria mediante adaptaciones curriculares y grupos flexibles como instrumento de atención a la diversidad. Las consecuencias de esta iniciativa es una respuesta general hacia una reducción esencialista del currículo (Aguado y Malik, 2001), acompañada de unas bajas expectativas del profesorado hacia el alumnado de minorías culturales (Jordán, 1994) que se manifiesta, en estudios de casos, desde la etapa infantil y que afecta especialmente al currículo científico (Cabo et al., 1996). Esta actitud del profesorado, junto a la escasez de materiales sobre ciencia intercultural (Membiela, 1999), pone la formación del profesorado de ciencias en el centro del problema, en el sentido de que el debate abierto en la comunidad científica de investigadores llegue también a la comunidad de enseñantes.

\section{CONCLUSIONES: PAPEL DE LA CIENCIA IN- TERCULTURAL EN EL FUTURO DE LA ENSE- NANZA DE LAS CIENCIAS}

De acuerdo con la bibliografía consultada, creemos que el concepto de ciencia intercultural puede dejar de ser un tópico marginal en el conjunto de la didáctica de las ciencias experimentales si se aceptan determinados marcos teóricos de referencia que le sirven de justificación. 
A. Desde el punto de vista de la naturaleza epistemológica de la ciencia, la ciencia intercultural implica asumir alternativas al positivismo, en el sentido de una ciencia tentativa o ciencia posnormal.

$B$. Con respecto al modelo de ciencia, implica la aceptación de las influencias culturales en la producción científica y viceversa, es decir, modelos de ciencia como práctica sociocultural.

$C$. Desde el punto de vista del que aprende, implica enfoques de constructivismo social, frente a versiones más individuales y cognitivas. Las experiencias y concepciones espontáneas de los sujetos tienen un origen social y, por tanto, tomar en consideración sus ideas como punto de partida invita a tomar igualmente como punto de partida para el proceso enseñanza-aprendizaje el origen cultural del alumnado.

$D$. En el ámbito didáctico, plantea el problema de la convivencia de tres clases de ciencias: personal, escolar y como ciencia occidental, y de la relación de cada una de ellas con sus correspondientes escenarios.

E. Como desarrollo curricular, implica una orientación centrada en el que aprende más que en la propia estructura de la materia. Y más que centrada en el que aprende, en el escenario sociocultural del que aprende, al concentrarse los problemas en centros urbanos y aumentar su conflictividad con el gradiente centro - periferia urbana.

$F$. Desde el punto de vista ideológico, implica la defensa de una serie de valores de justicia social, solidaridad, tolerancia e igualdad de oportunidades a favor de los más necesitados, y de ser conscientes de la politización intrínseca del currículo.

\section{REFERENCIAS BIBLIOGRÁFICAS}

AGUADO, T. y MALIK, B. (2001). Cultural diversity and school equality: Intercultural education in Spain from a european perperctive. Intercultural Education, 12(2), pp. 149-161.

AHLGREN, A. (1996). Comments on «multiculturalism, universalism and Science Education». Science Education, 80(3), pp. 361-363.

AIKENHEAD, G.S. (1993). Foreword: Multicultural issues and perpectives on Science Education. Science Education, 77(6), pp. 659-660.

AIKENHEAD, G.S. (1997). Toward a first nations crosscultural science and technology curriculum. Science Education, 81 pp. $217-238$.

AIKENHEAD, G.S. (2001). Students' ease in crossing borders into school science. Science Education, 85(2), pp. 180-188.
$G$. Todo ello creemos que encaja en una orientación CTS: una finalidad centrada en la formación de ciudadanos democráticos iguales y solidarios, en contenidos sobre ciencia y no sólo de ciencia, con inclusión de filosofía, historia, ética, epistemología... y en una contextualización de la ciencia abierta a las complejas influencias con la sociedad, el medio ambiente y la tecnología, especialmente con sus consecuencias, que también deben formar parte del currículo, incluyendo los debates y dilemas como contenido de enseñanza y no sólo conocimiento científico consensuado.

La ciencia intercultural, en sus diversas orientaciones, será un tópico central en la didáctica de las ciencias experimentales si los investigadores y profesores se guían por un conjunto de fundamentos teóricos que hemos apuntado aquí y que deberán desarrollarse y matizarse en el futuro. La definición de ciencia intercultural como movimiento, al igual que la orientación CTS, supone que el éxito o fracaso de las propuestas se mide por su capacidad de sumar voluntades al movimiento de reforma y, en definitiva, por el desarrollo de las agendas de trabajo marcadas, la multiplicación de los grupos de trabajo, la creación de redes de investigadores, boletines, revistas y reuniones científicas propias.

La existencia de una tradición europea y otra norteamericana sobre la cuestión no debe interpretarse desde el punto de vista del bien y del mal, sino desde la complejidad del problema. El contexto multicultural es una tendencia firme en todas las sociedades, pero provoca escenarios socioculturales, o comunidades de prácticas muy diversas, de forma que no se trata de elegir entre una tradición u otra sino en la utilidad de las propuestas para un escenario concreto. De la misma manera que las personas, ideas, revistas y libros cruzan el Atlántico, así lo hacen igualmente los problemas a los que cada tradición se ha dedicado.

AIKENHEAD, G.S. y JEGEDE, O.J. (1999). Cross-cultural Science Education: A cognitive explanation of a cultural phenomenon. Journal of Research in Science Teaching, 36(3), pp. 269-287.

AKATUGHA, A.H. y WALLACE, J. (1999). Sociocultural influences on physics students' use of proportional reasoning in a non-western country. Journal of Research in Science Teaching, 36 (3), pp. 305-320.

ALLEN, N.J. y CRAWLEY, F.E. (1998). Voices from the bridge: worldview conflicts of Kickapoo students of Science. Journal of Research in Science Teaching, 35(2), pp. 111-132.

ATWATER, M.M. (2000). Equity for Black Americans in precollege science. Science Education, 84, pp. 154-179.

ATWATER, M.M. y RILEY, J. (1993). Multicultural Science 
Education: Perspectives, definitions and research agenda. Science Education, 77, pp. 661-668.

ATWATER, M.M., WIGGINS, J. y GARDNER, C. (1995). A study of urban middle school students with high and low attitudes toward science. Journal of Research in Science Teaching, 32(6), pp. 665-675.

BAKER, D. y TAYLOR, P.C.S. (1995). The effect of culture on the learning of science in non-western countries: The results of an integrated research review. International Journal of Science Education, 17(6), pp. 695-704.

BARTOLOMÉ, M. (1997). Panorámica general de la investigación sobre educación multicultural en Europa. Revista de Investigación Educativa, 15(1), pp. 7-28.

BARTON, A.C. y YANG, K. (2000). The culture of power and Science Education: Learning from Miguel. Journal of Research in Science Teaching, 37(8), pp. 871-889.

BENARROCH, A. (2001). Interculturalidad y enseñanza de las ciencias. Alambique, 29, pp. 9-23.

BIANCHINI, J.A., HILTON-BROWN, B.A. y BRETON, T.D. (2002). Professional development for university scientists around issues of equity and diversity: Investigating dissent within community. Journal of Research in Science Teaching, 39(8), pp. 738-771.

BIANCHINI, J.A. y SOLOMON, E.M. (2003). Constructing views of science tied to issues of equity and diversity: A study of beginning science teachers. Journal of Research in Science Teaching, 40(1), pp. 53-76.

BROWN-ACQUAYE, H.A. (2001). Each is necessary and none is redundant: The need for science in developing countries. Science Education, 85, pp. 68-70.

CABO, J.M. et al., (1996). Intercultura e igualdad de oportunidades. Aldaba, 27, pp. 93-103.

CABO, J.M. y ENRIQUE, C. (2001). Ciencia intercultural. Epistemología e historia de la ciencia, en Actas del Congreso Nacional de Didácticas Específicas. Las Didácticas de las Áreas Curriculares en el siglo XXI Vol. I, pp. 335-344.

CABO, J.M. y ENRIQUE, C. (2003). Las relaciones ciencia, tecnología y sociedad en contexto multicultural, en Actas de las II Jornadas de Intercambio de Experiencias Educativas, Melilla, 28-30 de noviembre de 2001, pp. 35-44.

CALLON, M. (2001). Cuatro modelos de dinámica de la ciencia, en Ibarra, A. y López, J.A. (eds.). Desafíos y tensiones actuales en ciencia, tecnología y sociedad, pp. 27-69. Madrid: Biblioteca Nueva.

CHINN, P.W.U. (2002). Asian and Pacific islander women scientifics and engineers: A narrative exploration of model minority, gender and racial estereotipes. Journal of Research in Science Teaching, 39(4), pp. 302-323.

CLERK, D. y RUTHERFORD, M. (2000). Language as confounding variable in the diagnosis of misconceptions. International Journal of Science Education, 22(7), pp. 703-717.

COBERN, W.W. (1996). Constructivism and non-western science education research. International Journal of Science Education, 18(3), pp. 295-310.
COBERN, W.W. y LOVING, C.C. (2001). Defining «Science» in a multicultural world: Implications for science education. Science Education, 85, pp. 50-67.

DAVIS, K.S. (2001). «Peripheral and subversive»: Women making connections and challenging the boundaries of the science community. Science Education, 85, pp. 368-409.

DAVIS, K.S. (2002). Advocating for equitable science-learning opportunities for girls in an urban city youth club and the Roadblocks faced by women science educators. Journal of Research in Science Teaching, 39(2), pp. 151-163.

DURAN, B.J., DUGAN, T. y WEFFER, R. (1998). Language minority students in high school: The role of language in learning Biology concepts. Science Education, 82, pp. 311-341.

EIDE, K.Y. y HEIKKINEN, M.W. (1998). The inclusion of multicultural material in middle school science Teachers' resource manuals. Science Education, 82, pp. 181-195.

ENRIQUE, C. y CABO, J.M. (en prensa). Ciencia en contexto multicultural. Relaciones Ciencia / Religión y sus implicaciones en la enseñanza de las ciencias, en Actas del Congreso Internacional La Ciencia ante el público. Cultura humanista y desarrollo científico-tecnológico. Salamanca, 28-31 de octubre de 2002.

FARENGA, S.J. y JOYCE, B.A. (1999). Intentions of young students to enroll in science courses in the future: An examination of gender differences. Science Edcuation, 83(1), pp. $55-75$.

FELTHAM, N.F. y DOWNS, C.T. (2002). Three forms of assessment of prior knowledge, and improved perfomance followind an enrichment programme of English second language biology students within the context of a marine theme. Internacional Journal of Science Education, 24(2), pp. 157-184.

GONZÁLEZ, M. (2001). ¿Hacia donde dirigir la mirada? La reflexividad desde la perspectiva del género, en Ibarra, A. y López, J.A. (eds.). Desafíos y tensiones actuales en ciencia, tecnología y sociedad, pp. 119-134. Madrid: Biblioteca Nueva.

GONZÁLEZ, M., LÓPEZ, J.A. y LUJÁN, J.L. (1996). Ciencia, tecnología y sociedad: una introducción al estudio social de la ciencia y la tecnología. Madrid: Tecnos.

GOOD, R. (1995). Comments on multicultural Science Education. Science Education, 79(3), pp. 335-336.

HODSON, D. (1993). In search of a rationale for multicultural Science Education. Science Education, 77(6), pp. 685-711.

HODSON, D. (1999). Going beyond cultural pluralism: Science Education for sociopolitical action. Science Education, 83, pp. 775-796.

IRZIK, G. (2001). Universalism, multiculturalism and science education. Science Education, 85, pp. 71- 73.

JAYARATNE, T.E., THOMAS, N.G. y TRAUTMANN, M. (2003). Intervention program to keep girls in the science pipeline: Outcome differences by ethnic status. Journal of Research in Science Teaching, 40(4), pp. 393-414.

JEGEDE, O.J. (1997). School science and the development of scientific culture: A review of contemporany science educa- 
tion in Africa. International Journal of Science Education, 19(1), pp. 1-20.

JEGEDE, O.J. y OLAJIDE, J.O. (1995). Wait-time, classroom discourse and the influence of sociocultural factors in science teaching. Science Education, 79 (3) pp. 233-249.

JORDÁN, J.A. (1994). La escuela multicultural. Un reto para el profesorado. Barcelona: Paidós.

KAWAGLEY, A.O., NORRIL-TULL, D. y NORRIS-TULL, R.A. (1998). The indigenous worldview of Yupiang culture: Its scientific nature and relevance to the practice and teaching of science. Journal of Research in Science Teaching, 35(2), pp. 133-144.

KEARSEY, J. y TURNER, S. (1999). The value of bilingualism in pupils' understanding of scientific language. International Journal of Science Education, 21 (10), pp. 1037-1050.

KRUGLY-SMOLSKA, E. (1995). Cultural influences in science education. International Journal of Science Education, 17 , pp. $45-58$

KRUGLY-SMOLSKA, E. (1996). Scientific culture, multiculturalism and the science classroom. Science \& Education, 5, pp. 21-29.

KRUGLY-SMOLSKA, E. (1997). Science education in an evolving multicultural education, en McLeod, K. (ed.). Multicultural education. The challenges and the future. Winnipeg Canadian Association of Second Language Teachers.

LEMKE, J.L. (1997). Aprender a hablar ciencia. Lenguaje, aprendizaje y valores. Barcelona: Paidós.

LEMKE, J.L. (2001). Articulating communities: Sociocultural perpectives on Science Education. Journal of Research in Science Teaching, 38(3), pp. 296-316.

LEE, O. (1997). Diversity and equity for Asian-american students in Science Education. Science Education, 81, pp. 107-122.

LEE, O. (2001). Culture and language in Science Education: What do we know and what do we need to know? Journal of Research in Science Teaching, 38(5), pp. 499-501.

LEWIS, B.F. y AIKENHEAD, G.S. (2001). Introduction: Shifting perspectives from universalism to cross-culturalism. Science Education, 85, pp. 3-5.

LOVING, C.C. (1995). Comment on «multiculturalism, universalism and Science Education». Science Education, 79(3), pp. 341-348

LUBBEN, F., CAMPBELL, B. y DLAMINI, B. (1996). Contextualizing science teaching in Swaziland: Some student reactions. International Journal of Science Education, 18(3) pp. 311-320.

LUBBEN, F., NETSHISAULU, T. y CAMPBELL, B. (1999). Students' use of cultural metaphors and their scientific understandings related to heating. Science Education, 83, pp. 761-774.

LUFT, J., BRAGG, J. y PETERS, C. (1999). Learning to teach in a diverse setting: A case study of a multicultural Science Education enthusiast. Science Education, 83, pp. 527-543.
LYNCH, P.P. (1996). Students' alternative frameworks of matter: A cross-cultural study of linguistic and cultural interpretations. International Journal of Science Education, 18 (6), pp. 743-752.

LYNCH, S. (2001). «Science for all» is not equal to «One Size fits all»: Linguistic and cultural diversity and Science Education Reform. Journal of Research in Science Teaching, 38(5), pp. 622-627.

MANASSERO, M.A. y VÁZQUEZ, A. (2003). Los estudios de género en la enseñanza de las ciencias. Revista de Educación, 330, pp. 251-280.

MATTERN, N. y SCHAU, C. (2002). Gender differences in science attitude-achievement relationships over time among while middle-school students. Journal of Research in Science Teaching, 39(4), pp. 324-340.

McCARTHY, C. (1994). Racismo y currículum. Madrid: Morata.

McKINLEY, E. (2001). Cultural diversity: Masking power with innocence. Science Education, 85, pp. 74-76.

MEMBIELA, P. (1999). La enseñanza multicultural de las ciencias experimentales. Alambique, 22, pp. 117-122.

MUÑOZ, A. (1997). Educación intercultural. Teoría y práctica. Madrid: Escuela Española.

NINNES, P. (2000). Representations of indigenous knowledges in secondary school science textbooks in Australia and Canada. International Journal of Science Education, 22(6), pp. 603-617.

OGUNNIYI, M.B. (1995). The development of Science Education in Botswana. Science Education, 79(1), pp. 95-109.

ORTIZ DE MONTELLANO, B.R. (2001). Multicultural science: Who benefits? Science Education, 85, pp. 77-79.

OGAWA, M. (1995). Science Education in a multiscience perpective. Science Education, 79(5), pp. 583-585.

PORLÁN, R., RIVERO, A. y MARTÍN, R. (2000). Conocimiento del profesorado sobre la ciencia, su enseñanza y aprendizaje, en Perales, J. y Cañal, P. (dirs.). Didáctica de las Ciencias Experimentales, pp. 507-533. Alcoy: Marfil.

RAKOW, S.J. y BERMÚDEZ, A.B. (1993). Science is «Ciencia»: Meeting the needs of Hispanic-American students. Science Education, 77 (6), pp. 669-683.

RENNIE, L.J. y DUNNE, M. (1994). Gender, ethnicity and students' perceptions about science and sce-related careers in Fiji. Science Education, 78(3), pp. 285-300.

RODRÍGUEZ, A. J. (1997). The dangerous discourse of invisibility: a critique of the National Research Council's National Science Education Standars. Journal. of Research in Science Teaching, 34(1), pp. 19-37.

RODRÍGUEZ, A.J. (1998). Strategies for counterresistance: Toward socialtransformative constructivism and learning to teach Science for diversity and for understanding. Journal of Research in Science Teaching, 35(6), pp. 589-622.

ROTH, W.M. y LAWLESS, D. (2002). Science, culture and the emergence of language. Science Education. 86(3), pp. $368-385$. 
ROTH, W.M. y WELZEL, M. (2000). From activity to gestures and scientific language. Journal of Research in Science Teaching, 38(1), pp. 103-136.

SALES, I. y GARCÍA, R. (1997). Programas de educación intercultural. Bilbao: Deslclée de Brouwer.

SEILER, G., TOBIN, K. y SOKOLIC, J. (2001). Design, technology and science: Sites for learning, resistanse and social reproduction in urban schools. Journal of Research in Science Teaching, 38(7), pp. 746-767.

SMITH, F.M. y HAUSAFUS, C.O. (1998). Relations of family support and ethnic minority students' achievement in science and mathematics. Science Education, 82, pp. 111-125.

SNIVELY, G. y CORSIGLIA, J. (2001). Discovering indigenous science: Implications for science education. Science Education, 85, pp. 6-34.

SORIANO, E. (1997). Análisis de la educación multicultural en los centros educativos de la comarca del poniente almeriense. Revista de Investigación Educativa, 15(1), pp. 43-67.

STANLEY, W.B. y BRICKHOUSE, N. W. (1994). Multiculturalism, universalism and Science Education. Science Education, 78(4), pp. 387-398.

STANLEY, W.B. y BRICKHOUSE, N.W. (2001). Teaching sciences: The multicultural question revisited. Science Education, 85 , pp. $35-49$.

SUTHERLAND, D. y DENNICK, R. (2002). Exploring culture, language and the perception of the nature of science. International Journal of Science Education, 24 (1), pp. 1-25.

SVENNBECK, M. (2001). Rethinking the discusión about science education in a multicultural world: Some alternative questions as a new point of departure. Science Education, 85 , pp. $80-81$.

TSAI, C. (1998). An analysis of scientific epistemological beliefs and learning orientations of Taiwanese eighth graders. Science Education, 82, pp. 473-489.

UNSWORTH, L. (2001). Evaluating the language of different types of explanations in junior high school science texts. International Journal of Science Education, 23(6), pp. 585-609.

WALDRIP, B.G. y TAYLOR, P.C. ( 1999a). Standars for cultural contextualization of interpretative research: A Melanesian case. International Journal of Science Education, 21(3), pp. 249-260.

WALDRIP, B.G. y TAYLOR, P.C. (1999b). Permeability of students' worldviews to their school views in a non-western developing country. Journal of Research in Science Teaching, 36(3), pp. 289-303.

WILLIAMS, H. (1994). A critique of Hodson's «in Search of a rationale for multicultural science education». Science Education, 78(5), pp. 515-519.

ZEMBYLAS, M. (2002). The global, the local and the Science curriculum: A strugge for balance in Cyprus. International Journal of Science Education, 24(5), pp. 499-519. 